\title{
Duchenne muscular dystrophy: CRISPR/Cas9 treatment
}

\author{
Cell Research (2016) 26:513-514. doi:10.1038/cr.2016.28; published online 1 March 2016
}

\begin{abstract}
A novel approach to gene correction by genome editing shows great promise as a treatment for Duchenne muscular dystrophy (DMD). CRISPR/Cas9 delivered by adenoassociated virus to a mouse model for DMD demonstrated improvement in function and histology.
\end{abstract}

Duchenne muscular dystrophy (DMD) is the most common severe form of childhood muscular dystrophy affecting 1:5 000 newborn males [1]. Both skeletal and cardiac muscles are affected. If untreated, loss of ambulation is typically seen in 10-12 years. Death occurs in early to mid-twenties due to heart failure. Only a single class of pharmacologic intervention has been shown to increase muscle strength in DMD patients. The documented benefit of corticosteroids was demonstrated in a randomized, placebo-controlled trial of prednisone [2] and validated subsequently in multiple controlled studies [3]. Corticosteroid treatment can result in 1-2 years of prolongation of ambulation but the side effect profile limits its use, which includes weight gain, cushingoid appearance, osteoporosis, cataracts, and glucose intolerance. Deflazacort, an alternative glucocorticoid, is equally effective with slightly less weight gain [4]. Treatment directed toward correcting the underlying molecular defect can be achieved by skipping exon 51 of the $D M D$ gene using eteplirsen, a morpholino-based oligonucleotide. Studies over 4 years show prolonged ambulation and change in the rate of decline compared to agematched controls measured by the distance walked using the six minute walk test [5]. Other treatments in clinical trial include upregulation of utrophin using a drug (SMT C1100) that delivers a small molecule to increase production of utrophin in pre-clinical studies [6]. The drug is well tolerated and safe and its efficacy is being tested in a Phase 2 clinical trial. Gene replacement therapy is also underway, but problems arise because the $D M D$ gene is too large to be packaged in adeno-associated virus (AAV). Therefore, a mini-/microdystrophin (4-5 kb) with diminished function must replace the full-length cDNA ( $>$ $11 \mathrm{~kb})$. We have completed one study delivering minidystrophin in AAV. We found only minimal gene expression and demonstration of a $\mathrm{T}$ cell response when we expressed minidystrophin in a deleted region of the patient's $D M D$ gene [7].

A novel approach to gene correction by genome editing shows great promise for clinical translation, highlighted by recent reports from three separate laboratories in Science [8-10]. In all three studies, the CRISPR/Cas9 system targeted the point mutation in exon 23 of the $\mathrm{mdx}$ mouse that creates a premature stop codon and serves as a representative model of DMD. These studies are important in multiple ways. First, they provide a path, laying the groundwork for clinical translation addressing many of the critical questions that have been raised regarding this system of gene correction. Each of the three studies demonstrated efficacy using a two-vector system of AAV-CRISPR rather than single vectors for both the guide RNA and the Cas9 nuclease. The cDNAs from Staphylococcus aureus Cas9 and Streptococcus pyogenes Cas 9 were both effective in these in vivo pre-clinical studies and delivery of vectors using either AAV serotypes, AAV9 or AAV8, performed well. Prior clinical studies using these AAV serotypes have proven safe, further demonstrating the feasibility for human application. There was general agreement between laboratories on restoration of dystrophin-positive fibers. Importantly, functional recovery was demonstrated in CRISPR/Cas9treated mice, including increased grip strength, improved force generation, resistance against eccentric contraction, and reduced serum creatine kinase (CK). Histologically, there was reversal of muscle necrosis, fewer infiltrating inflammatory cells, and decreased fibrosis. Immune staining showed the restoration of nNOS activity and components of the dystrophin-glycoprotein complex. Of particular interest clinically, dystrophin expression was seen in vascular smooth muscle, an important source of oxygenation during muscle activity. In all three studies, cardiomyocytes demonstrated the restoration of dystrophin. The importance of improving cardiac function cannot be overlooked clinically because dystrophin deficiency in the heart is a common mode of death for the DMD patient [11].

The studies are also credited with relieving some of the anxiety expressed regarding the CRISPR/Cas9 approach. Genome editing has repeatedly been charged with the concerns of off-target effects. All three studies explored deep sequencing of gRNA target sites with specific attention to the top 10 predicted possibilities. None of the studies showed evidence of off-target gene editing that would be of concern clinically and CRISPR-treated mice showed no 
phenotypic evidence of toxicity.

An important finding emerging from one of the reports, the Wagers' study [10], was the demonstrated efficiency of editing of Pax7-positive muscle satellite cells. This finding establishes the potential for gene correction of dystrophic myofibers that have been damaged followed by replacement of dystrophin by repopulated satellite cells expressing the missing protein. Over time this will enhance recovery of dystrophic muscle and improve clinical function.

Overall the path to recovery using genome editing with CRISPR/Cas9 delivered by AAV is poised for translation and looks very promising. Once proof of principle is established using CRISPR/ Cas9 editing of humanized mutations in DMD animal models, the path to clinic will move quickly. It has been estimated that CRISPR-mediated removal of one or more exons from the genomic DNA could benefit as many as $80 \%$ of DMD patients. Furthermore, gene editing at an early age of intervention would be the ideal scenario, before waves of necrosis lead to muscle fiber loss, replacement of tissue by fat, and fibrosis. We know from newborn screening studies that the disease is present at birth given that serum CK has already reached 10 times normal level. There is also a proven method of early detection validated throughout the State of Ohio in nearly 40000 male births that will hopefully soon be adopted for a wider application [1]. Merging CRISPR/Cas9 treatment early in life with newborn screening could be one answer to a devastating disease of childhood.

Jerry R Mendell ${ }^{1,2,3}$, Louise R Rodino-Klapac ${ }^{1,2,3}$

${ }^{1}$ Department of Pediatrics, ${ }^{2}$ Center for Gene Therapy, Nationwide Children's Hospital, ${ }^{3}$ The Ohio State University, Columbus, OH 43205, USA Correspondence: Jerry R Mendell
E-mail: Jerry.Mendell@nationwidechildrens.org

\section{References}

1 Mendell JR, Shilling C, Leslie ND, et al. Ann Neurol 2012; 71:304-313.

2 Mendell JR, Moxley RT, Griggs RC, et al. $N$ Engl J Med 1989; 320:1592-1597.

3 Griggs RC, Herr BE, Reha A, et al. Muscle Nerve 2013; 48:27-31.

4 Biggar WD, Harris VA, Eliasoph L, et al. Neuromusc Disord 2006; 16:249-255.

5 Mendell JR, Goemans N, Lowes LP, et al. Ann Neurol 2016; 79:257-271.

6 Tinsley J, Robinson N, Davies KE. J Clin Pharmacol 2015; 55:698-707.

7 Mendell JR, Campbell K, Rodino-Klapac L, et al. $N$ Engl J Med 2010; 363:1429-1437.

8 Long C, Amoasil L, Mireault AA, et al. Science 2016; 351:400-403.

9 Nelson CE, Hakim CH, Ousterout DG, et al. Science 2016; 351:403-407.

10 Tabebordbar M, Zhu K, Cheng JK, et al. Science 2016; 351:407-411.

11 McNally EM, Kaltman JR, Benson DW, et al. Circulation 2015; 131:1590-1598. 